\title{
Zum Problem der ,poturice“ in Montenegro
}

\author{
Hans-Michael Miedlig
}

Die sog. ,poturice“, d.h. die ehemals christliche, nach der osmanischen Eroberung des Balkans jedoch zum Islam übergetretene einheimische Bevölkerung, ist bisher in der Forschung überwiegend im Zusammenhang mit den Verhältnissen in Bosnien und der Herzegowina behandelt worden. Dies erscheint einleuchtend, hat die Islamisierung doch gerade hier, wie es Franz Babinger einmal ausdrückte, ,eine wirkliche Tiefenwirkung“" erzielt ${ }^{1}$ und die Gesellschaft in weiten Teilen grundlegend transformiert. Was Montenegro betrifft, so blieb der Islam jedoch keineswegs - um noch einmal Babinger zu zitieren - lediglich ,firnisartig an der Oberfläche haften". ${ }^{2}$ Davon zeugt schon der für das 16. bis ins 19. Jahrhundert dort belegte Familienname „Poturica“. ${ }^{3}$ Dennoch hat selbst die historische und ethnologische Forschung der montenegrinischen islamisierten Bevölkerungsgruppe bislang nur wenig Aufmerksamkeit gewidmet. ${ }^{4}$ Der Gegenstand wurde eher dann und wann von der Slawistik im großen Zusammenhang mit dem bedeutenden Epos des montenegrinischen Dichterfürsten Petar Petrović II. Njegoš Der Bergkranz wahrgenommen. ${ }^{5}$ In diesem Werk wird das Thema der sog. „Vernichtung der Poturice“ (Istraga poturica) verarbeitet. Njegoš lehnt sich dabei an ein als Massaker historisch nicht belegtes und deshalb eher als Legende einzustufendes Ereignis ${ }^{6}$ aus der Regierungszeit des Bischofs Danilo Šćepčev Petrović, des Begründers der Njegoši-Dynastie, zu Beginn des 18. Jahrhunderts an. Auf jenes Geschehnis bzw. seine Überlieferung wird im Folgenden noch zurückzukommen sein.

Die wörtliche Übersetzung des serbischen bzw. kroatischen Wortes ,,poturica" ist nicht ganz einfach, wenn alle Facetten seiner Semantik adäquat berück-

1 Babinger 1959, 205.

2 Ebd.

3 Rječnik 1935, 222.

4 Für die nachfolgende Analyse wird das Wenige, was an Literatur zum Gegenstand existiert bzw. zugänglich ist, berücksichtigt. Das sehr seltene Büchlein von M. Dragović 1931 steht dem Verfasser dieses Aufsatzes leider nicht zur Verfügung. Es wurde später als Abschnitt in ein größeres Werk desselben Autors eingearbeitet, vgl. Dragović 1935. Auch diese Darstellung kann wegen fehlender Zugänglichkeit hier nicht herangezogen werden.

5 Vgl. etwa Deretić 1996, 143-145, 151; Kalezić 1987, 133-135; Rizvić 1985, 8-13, 1633; Medenica 1964, 125-135.

6 So Dašić 1999, 113. Vgl. auch Bartl 1974, 370. 
sichtigt werden sollen. So verzeichnet das 1928 erschienene „Wörterbuch der serbokroatischen und der deutschen Sprache" von Svetomir Ristitsch (Ristić) und Jowan (Jovan) Kangrga in seinem 2. Teil unter „poturica“ „gewordener Türke, Aftertürke, Renegat". ${ }^{7}$ Mehr noch hat diese Semantik Vuk Stefanović Karadžić in seinem berühmten serbischen Wörterbuch hervorgehoben, wo er „unechter Türke, Aftertürke, pseudoturca“ notiert und in Kenntnis des damaligen Sprachgebrauchs der orthodoxen Montenegriner vor „unechter Türke“ noch in Klammern das Wort „verächtlich“ hinzusetzte. ${ }^{8}$ Die hier anklingende pejorative Tönung ist in der Tat integraler Bestandteil der Semantik. Sie entwikkelte sich in Montenegro besonders im Zusammenhang mit den zunehmenden Kämpfen der Stämme gegen den osmanischen Oberherrn bzw. den örtlichen paşa und seine Truppen zur Sicherung ihrer Autonomie seit der Wende vom 17. zum 18. Jahrhundert. Diese negative Haltung gegenüber den „poturice“ ergab sich einerseits aus der Tatsache, dass die Konvertiten den islamischen Machthabern oft genug, und zwar nicht nur in Kriegszeiten, als Brückenköpfe der Islamisierung dienten. ${ }^{9}$ So stabilisierten sie als - in den Augen vieler Stammesangehöriger - illoyale, unsichere Elemente den Zustand der permanenten Gefährdung der de facto autonomen Stammesgebiete (knežine) durch die osmanischen Machthaber der Region. Diese Gefährdung war zumindest zu einem großen Teil auch die Folge der zahlreichen bewaffneten An- und Übergriffe der Stämme auf die örtlichen osmanischen Garnisonen, worauf die Pforte mit teilweise verheerenden Strafexpeditionen reagierte, besonders in den Jahren 1712-1714. ${ }^{10}$ Auch die kompromisslos türkenfeindliche Politik des Bischofs Danilo (16961735), besonders in der ersten Phase seiner Herrschaft, hatte am Aufbau von Ressentiments gegenüber den ,poturice“ großen Anteil, da Danilo darauf zielte, diese islamisierte Bevölkerungsgruppe, für die allmählich auch die Bezeichnung „Türken“ üblich wurde, aus den Stammesverbänden zu drängen. ${ }^{11}$

So wurden die Bezeichnungen ,poturica“ und „turčin“ (,Türke“) zu negativ aufgeladenen Synonymen.

Eine spezielle Gruppe unter diesen hier thematisierten „Renegaten“, nämlich diejenigen, die sich nur nach außen unter dem Druck ihrer islamischen Um-

7 Ristitsch / Kangrga 1928, 732.

8 Karadžić 1898, 575.

9 Vgl. Vasić 1985, 12.

10 Gemeint sind die Feldzüge gegen die aufständischen montenegrinischen Stämme unter Führung des bosnischen Wesirs Ahmed Paşa (1712) sowie die großangelegte Strafexpedition unter Führung des Köprülü Numan Paşa (1714). Letztere verwüstete das Land in besonders verheerender Weise mit zahlreichen Opfern und Gefangennahmen auf beiden Seiten und zwang den regierenden Bischof (Danilo) vorübergehend ins Exil. Genaueres zu diesen Kleinkriegen bei Hadrijahić 1980, 85-90.

11 Istorija Crne Gore 1975, 246. 
gebung, gewissermaßen demonstrativ dem Islam als neuer Religion zuwandten, ansonsten aber diskret in ihrem Familienkreis ihren christlichen Glauben nach wie vor praktizierten und manchmal sogar heimlich in die Kirche gingen, sollen in den nachfolgenden Ausführungen nicht weiter berücksichtigt werden. Hier handelt es sich nämlich um Kryptochristen, die in Montenegro, in Mazedonien und Albanien, ferner auch in den anderen osmanisch beherrschten Balkangebieten mehr oder weniger stark vertreten waren. ${ }^{12}$ Offiziell mit allen Rechten und Vorrechten eines gläubigen Muslim ausgestattet, wurden sie jedoch durch ihren faktischen Verbleib in der christlichen Gemeinschaft nicht zu Außenseitern ihrer Sippe bzw. ihres Stammes. Diese Leute mit zweierlei Glauben, wie sie in einer Analyse zum islamisch-christlichen Synkretismus in den Ländern des ehemaligen Jugoslawiens genannt werden, stellen ein Spezialproblem dar, das deshalb auch in einem gesonderten Aufsatz behandelt werden sollte. ${ }^{13}$

Die muslimische Schicht der sog. ,poturice“ in Montenegro entstand im wesentlichen Ende des 16. und blühte im 17. Jahrhundert durch massenhafte Konversionen geradezu auf. Sie wuchs zeitlich, regional und quantitativ ungleichmäßig besonders in den Städten sowie den Dörfern der Ebenen. ${ }^{14} \mathrm{Ge}-$ rade hier hatten sich zahlreiche ethnische Türken angesiedelt, auf dem Land nicht zuletzt die Sipahi als zunehmend in privater Verantwortung wirtschaftende Steuerpächter und Herren über die Cifflik-Güter mit einer großen Zahl politisch und ökonomisch minderberechtigter christlicher Bauern. Der Prozess der massenhaften Islamisierung betraf zunächst die Städte, bis zur Mitte des 17. Jahrhunderts war in allen städtischen Siedlungen des montenegrinischen Gebiets eine muslimische Mehrheit gewachsen. In den östlichen und nördlichen Randgebieten, die erst 1878 bzw. 1912 zu Montenegro kamen, also z.B. im oberen Limund Bihorgebiet, gab es häufige Konversionen zum Islam erst in der 2. Hälfte des 17. Jahrhunderts. Hier spielte äußerer Zwang durchaus eine Rolle. Nach gewaltsamen Übergriffen der osmanischen Behörden gegenüber der christlichen Bevölkerung in der serbisch-orthodoxen Metropolitandiözese Budimlja im sog. „kandischen Krieg“" (1645-1669) suchte manch einer Sicherheit im Islam. ${ }^{15}$

Auch die montenegrinischen Bergstämme wurden zumindest teilweise, je nach Siedlungsort und der Nähe eines muslimischen Milieus, von der Islamisierung erfasst, allerdings setzte der Prozess später ein, d.h. vom Ende des 17. bis ins 19. Jahrhundert. Hier tut man gut daran, die Verhältnisse differenziert zu

12 Vasić 1985, 11.

$13 \mathrm{Zu}$ diesen Angehörigen ,zweierlei Glaubens“ vgl. besonders Zirojević 2003, zu Montenegro hier 19, 35-37, 55; zum auch nach außen praktizierten Doppelglauben des formell islamisierten Stammes der Mrkovići im montenegrinischen Küstenland ebd., 41, 52.

14 Rastoder 2003, 114; Vasić 1985, 7; Clewing 1999, 482.

15 Dašić 1980, 44, 45. 
betrachten, denn einige Stämme, z.B. die strenggläubig orthodoxen Šekularci, die schon infolge geographischer Nähe zum Pećer Patriarchat zu diesem stabile Beziehungen unterhielten, entzogen sich dauerhaft einer Islamisierung. Dies gilt auch für nicht oder teilweise im Laufe der Zeit eigenstammlich organisierte, z.T. aber auch allmählich von den Vasojevići assimilierte Sippenverbände, die in den Quellen als Ašani bzw. Srbljaci auftauchen. Bei diesen handelt es sich um orthodoxe Alt- und Neusiedler verschiedenen ethnischen Ursprungs im oberen Lim-Gebiet, die sich dem nach 1690 dort zuwandernden Stamm der Vasojevići zwar in ihrer Gesellschaftsverfassung anglichen, aber dadurch abgrenzten, dass sie ihre Herkunft nicht von Vasa als gemeinsamem Stammvater ableiteten. ${ }^{16}$ Andere, etwa Teile der Vasojevići, Kuči, Bratonožići, Rovčani, Piperi, Bjelopavlići, besonders diejenigen, die in die Ebene des oberen Lim zuwanderten, wandten sich dem Islam ebenso zu, wie vom Osten einwandernde albanische Stämme der Hoti, Grude und Klimente, letztere häufig Katholiken, nun gleichfalls ,,poturice“. Wichtige Verbreitungszentren des Islam waren unter anderen die kompakten muslimischen Siedlungen Plav, Gusinje und Rožaje, die bis 1912 außerhalb des montenegrinischen States lagen und besonders auf die Bevölkerung am oberen Lim bis Bijelo Polje sowie ins benachbarte Bihor einwirkten. Der hier dominante Stamm der Vasojevići war, so ein ernstzunehmender montenegrinischer Historiker, „,bis in die dreißiger Jahre des 19. Jahrhunderts [...] durchsetzt mit ,poturice ““. ${ }^{17}$

Warum wagten montenegrinische orthodoxe, aber auch römisch-katholische Christen den folgenschweren Schritt der Trennung von der christlichen Wertegemeinschaft und traten zum Islam über, was verstärkt seit dem 18. Jahrhundert in den Augen ihrer nicht konversionswilligen christlichen Stammesbrüder die enge gesellschaftliche und geistige Verbindung mit den feindlichen Autoritäten bedeutete? Ein wichtiger Grund ist natürlich der unmittelbare Zwang, wie er in den Anfängen noch in dem devşirme, der Knabenlese, gegeben war. Hiervon abgesehen existierte in der Praxis ein System von direkten und indirekten Zwängen, das zu einer vom Betroffenen manchmal mehr oder weniger akzeptierten Islamisierung führte. Hier ist z.B. die häufig vorkommende Verführung von Sippenmitgliedern durch bereits islamisierte Verwandte oder Nachbarn zu nennen. Sprach ein Christ die Kelime-i Şehadet einfach nur einmal nach, die lautet: „Ich

16 Vgl. ebd., 18f. Zur historischen Bevölkerungsverteilung, Siedlungsgeographie und Migrationen in diesem Gebiet des heutigen Nordmontenegro nach wie vor wertvoll: Pejović 1979, $72 \mathrm{ff}$.

17 Dašić 1980, 46-48, 52, 53 (Zitat S. 52). Die Vasojevići waren zunächst im Gebiet um Lijeva Rijeka im heutigen Ostmontenegro ansässig, hatten jedoch seit Ende des 17. Jahrhunderts nördlich der Kom-Berge am Oberlauf des Lim ihr Hauptsiedlungsgebiet, vgl. dazu Näheres ebd., $8 \mathrm{ff}$. 
bezeuge, dass es keinen anderen Gott außer Allah gibt und dass Muhammed der göttliche Gesandte ist", ohne sich über die Folgen restlos im Klaren zu sein, so hatte er nun umgehend zum Islam überzutreten. Eine Weigerung bedeutete schwere Bestrafung oder Tod. ${ }^{18}$ Entscheidend wirkte sich hier die Normen- und Verhaltenskontrolle durch die muslimische Umgebung aus.

In Zeiten der Kriege des Sultans mit einer christlichen Macht war die Gefahr einer Zwangsislamisierung besonders groß, so etwa bei den Katholiken des Erzbistums Bar an der montenegrinischen Küste. Diese wurden während des kandischen Krieges bezichtigt, in verräterischer Absicht gegen ihren Oberherrn zu revoltieren, und vor die Alternative gestellt, entweder den Islam anzunehmen oder getötet zu werden. ${ }^{19}$ So entstanden in diesem Fall katholische „poturice“ wider Willen. Selbstverständlich war nach den religiösen Rechtsvorschriften des Şeriat die Konversion zum Islam eine grundsätzlich freiwillige Angelegenheit. Dies bestätigen die bisherigen Forschungsergebnisse, wonach die meisten Glaubensübertritte zum Islam auf dem Balkan - von der Knabenlese und Versklavung einmal abgesehen - durchaus in freier Entscheidung erfolgten. ${ }^{20}$ Dazu zählen die Übertritte im montenegrinischen Gebiet wie andernorts nach der osmanischen Eroberung, als ehemalige Feudalherren mit diesem Schritt versuchten, ihre soziale Stellung als privilegierte Grundbesitzer, aber auch als oft vermögende Händler und Kaufleute, dauerhaft zu erhalten. ${ }^{21}$ Dies gilt besonders für die zweite Hälfte des 15. Jahrhunderts. ${ }^{22}$

In Montenegro verlief dieser Prozess durch die relativ späte Inkorporierung des Gebiets durch die Osmanen zeitlich verzögert erst im 16. Jahrhundert. Einen typischen Fall stellen die islamisierten montenegrinischen Grundbesitzerfamilien der Mušovići und Hasanbegovići dar. Mindestens erstere, wahrscheinlich aber auch letztere, stammten vom mittelalterlichen christlichen Feudalherrn Joksim Vraneški, also aus dem Gebiet um Vraneš, ab. Zwei seiner drei Söhne etablierten mit ihrer Konversion eine neue muslimische Linie der Familie und ließen sich im unteren Kolašin-Gebiet nieder. Ihre Nachkommen stiegen gesellschaftlich weiter auf, die der Mušovići fungierten von 1714 bis 1877 als osmanische Stadtkommandanten von Kolašin. ${ }^{23}$

Die Führer der neuen muslimischen Familien, die ja, soweit landbesitzende Agas, auch Herrschaftsfunktionen sowohl über muslimische als auch über christ-

18 Vasić 1985, 10.

19 Ebd., 11.

20 Minkov 2004, 193.

21 Vasić 1985, 8. Die Händlerschicht unter diesen sozial und politisch privilegierten Bevölkerungsgruppen betont für die Gebiete des heutigen Nordmontenegro Mušović 1981a, 7.

22 Minkov 2004, 99.

23 Vgl. Mušović 1981b, 89-92. 
liche Bauern innehatten, begriffen sich in ihrer Mehrheit - nicht zuletzt zur Absicherung ihrer privilegierten sozialökonomischen Position - als „Türken“, wobei sie an der historischen Realität vorbei eine passende Herkunftslegende konstruierten. Dieses „Wir-Gefühl“ teilten indessen keineswegs die rangniederen islamisierten Bevölkerungsschichten, die sich ihrer südslawisch-christlichen Abkunft meist bewusst blieben. ${ }^{24}$

Generell waren der soziale und ökonomische Aufstieg in der muslimischen Gesellschaft, das Erreichen politischer Gleichstellung sowie in den Städten die Anziehungs- und Wirkkraft der muslimischen religiösen Hochkultur wichtige Anreize, zum freiwillig erklärten ,poturica“ zu werden. ${ }^{25}$ So übte die im Südosten des heutigen Montenegros gelegene, im 17. Jahrhundert bereits völlig muslimisch geprägte Stadt Ulcinj eine starke Sogwirkung auf die christliche Bauernbevölkerung der Umgebung aus. Ein nach Rom geschickter Bericht des katholischen Missionars Bruder Leonardi von 1640 erzählt, dass zu jener Zeit ein starker Zuzug von Christen aus dem näheren Umkreis in den genannten Ort stattgefunden habe und die Umsiedler nicht nur in türkische Dienste getreten, sondern auch nach und nach zum Islam konvertiert seien. ${ }^{26}$ Allerdings muss hier noch einmal ausdrücklich betont werden, dass hinter diesen freiwilligen Entscheidungen, zum ,poturica“ zu werden, ein durchaus zwiespältiger Kontext stehen konnte. In der Praxis bestimmten die individuelle Entscheidungsfindung eben auch Elemente der direkten oder indirekten Nötigung, etwa durch den Einfluss islamischer Geistlicher oder, wie oben schon angeführt, seitens der muslimischen Nachbarschaft auf den oder die betroffenen Christen. ${ }^{27}$ Auch die unmittelbar bevorstehende Gefahr der Umsetzung des alten Gewohnheitsrechts der Blutrache zuungunsten des Mitglieds eines verfeindeten Stammes ließ dieses zuweilen aus Schutzgründen zum Islam übertreten. ${ }^{28}$

Die negative Haltung der christlichen montenegrinischen Stämme und der serbisch-orthodoxen Kirchenorganisation gegenüber den Islamisierten schloss indessen nicht aus, dass Verbrüderungen sogar zwischen montenegrinischen orthodoxen Geistlichen mit bestimmten ,poturice“ häufig vorkamen. Im Stamm der Kuči, ansässig zwischen dem albanischen Stamm der Grude umweit von Podgorica im Süden, dem Kom-Gebirge im Norden, den Drekalovići im Westen sowie dem albanischen Stamm der Klimente im Osten war dies gängige Praxis. So verbrüderte sich der bekannte Stammesführer der Kuči und spätere Schriftsteller Marko Miljanov Popović mit den albanischen Konvertiten Şaban-beg aus

24 Ebd., 88, 89.

25 Clewing 1999, 482.

26 Mušović 1979, 69.

27 Vasić 1985, 9, 10.

28 Vgl. Pejović 1979, 72. 
Tuzi und Alil Beçova aus Tudjemil, ja selbst Petar Petrović II. Njegoš mit Alipaşa Stočević aus der Herzegowina. Diese Fälle können als Belege dafür gelten, dass die Stammesbräuche auch im 18. und 19. Jahrhundert im Bedarfsfall immer noch über die Gebote der serbischen Orthodoxie dominierten. Eine derartige Praxis ermöglichte etwa im unsicheren montenegrinisch-osmanischen Grenzgebiet Bewegungsfreiheit in beiden Richtungen und bot nicht zuletzt Schutz von Person und Besitz vor Angriffen lokaler osmanischer Amtsträger ${ }^{29}$, bediente letztlich also ein vitales Sicherheitsinteresse der montenegrinischen Stämme.

Es ist wichtig, hervorzuheben, dass die ,poturice“ mindestens bis zur Regierungszeit des Bischofs Danilo in der Regel friedlich mit ihren serbisch-orthodoxen Stammesbrüdern im gleichen Familien-, Bruderschafts- bzw. Stammesverband lebten. Religiöse Unterschiede spielten noch keine die Gemeinschaft spaltende Rolle. Das gewohnheitsrechtliche Stammesgesetz hatte Priorität, was auch hieß, dass durch den Übertritt zum Islam erworbene formale sozial-ökonomische Privilegien als innergesellschaftliche Konfliktpotentiale im praktischen Zusammenleben wenig Bedeutung hatten. ${ }^{30}$ Die genannten Verbrüderungen zwischen Christen und Konvertiten ergeben sich somit aus diesen Zusammenhängen. Zur Illustration des Gesagten passt das althergebrachte Stammesgesetz der Vasojevići. Dieses Gewohnheitsrecht wurde noch Ende 1829 oder in der ersten Hälfte des Jahres 1830 auf einer allgemeinen Stammesversammlung im Kloster Đurđevi stupovi, mit einigen Zusätzen versehen, bestätigt. Punkt 2. des Kodex sah vor, die ,,poturice“ keinesfalls zu töten, jedoch dafür zu sorgen, dass keine neuen Moscheen gebaut und die alten nicht vor dem Verfall bewahrt würden. Jeder Bruderschaft wurde es ins freie Belieben gestellt, ob sie ihre zum Islam konvertierten Mitglieder in den alten christlichen Glauben zurückführen wollten. Wenn jemand jedoch trotz dringender Aufforderung nicht zum alten Glauben zurückkehren mochte, waren die Stammesbrüder allerdings berechtigt, den Betreffenden mit Schlägen dazu zu zwingen. Die Konvertiten hatten zwar mit ihrem Glaubensübertritt zum Islam nun als „,Türken“ zu gelten, blieben aber dennoch weiter Stammesbrüder. Sie waren nach Punkt 9. des „Gesetzes“ frei und ungehindert in ihrer Religionsausübung, solange sie beim Beten auf ihrem eigenen Grund und Boden verblieben. Verletzten sie dabei jedoch die Grundstücksgrenze zu ihrem christlichen Nachbarn, so sollte dieser berechtigt sein, sie mit einer Stange zu verjagen und Prügel zu verabreichen. ${ }^{31}$

Diese bezüglich des Verhältnisses zwischen Muslimen und Christen innerhalb der Stammesgemeinschaft sehr heterogenen Bestimmungen, die den Muslim prinzipiell respektierten und gegen diesen nur unter ganz bestimmten Be-

29 Zirojević 2003, 56, 57.

30 Istorija Crne Gore 1975, 247.

31 Vasojevićki zakon 1929, 13, 14, 44. 
dingungen die Verhängung von Strafen oder gar Gewaltanwendung gestatteten, zeigt auch folgender Absatz der Bestimmung Nr. 9: Jedem christlichen Bewohner sollte es erlaubt sein, nach altem Brauch zur Abwehr teuflischer Einflüsse Holzkreuze in den Boden der den christlichen Grundbesitz umgebenden muslimischen Grundstücke zu rammen. Die ,poturice“ waren jedoch berechtigt, diese Kreuze wieder herauszureißen, durften dies aber erst am Folgetag nach Himmelfahrt tun, keinesfalls früher. Wer sich nicht an diese Frist hielt, musste dem Dorf eine Summe als Schadensersatz zahlen und dem Ortsvorsteher ein Mittagessen geben. ${ }^{32}$

Weitere Belege für die enge, wenn auch manchmal problematische Alltagsbeziehung zwischen den zum Islam Konvertierten und den beim alten Glauben gebliebenen Christen derselben Stammesbruderschaft liefern die frühen Feldforschungen des genannten Marko Miljanov. Die Ergebnisse der von Miljanov angestellten Umfragen bezüglich der im Land herrschenden Rechtsbräuche ergaben u.a., dass christliche Familien aus dem Stamm der Kuči im Osten des Landes ab und an ihre Töchter an Muslime verheirateten, was aus der Sicht der orthodoxen Kirche streng verboten, nach dem islamischen Eherecht jedoch erlaubt war. Dabei wurde verabredet, dass die Töchter als Ehefrauen der Muslime ihren alten Glauben auf Dauer beibehalten konnten und keinesfalls zur Annahme des Islams gezwungen werden sollten. Die aus solchen Ehen entspringenden Kinder hatten indessen als „Türken“ zu gelten. ${ }^{33}$ Dabei war es unerheblich, ob der Hausvorstand ein ethnischer Türke oder ein slawischer „poturica“ war. In der Praxis traten jedoch viele nichtmuslimische Ehefrauen aus nachvollziehbaren Gründen früher oder später dann doch zum Glauben ihres Mannes über. ${ }^{34}$

Ein weiteres Beispiel ist der Umgang der islamisierten Grundbesitzerfamilie der Ganići mit den ihnen zugeordneten christlichen Bauern des Dorfes Zagrađe im Umkreis von Bijelopolje. Die Ganići schützten und achteten die ererbte freie Selbstverwaltungstradition der Dorfbewohner und banden sie nicht in das sonst vorherrschende Ciftlik-System ein, da sie sich ihrer gemeinsamen Abkunft von den Kuči bewusst waren. ${ }^{35}$

Um das Bild bezüglich der tatsächlichen Stellung der islamisierten Bevölkerungsgruppe innerhalb der montenegrinischen Stammesgesellschaft abzurunden, ist es angebracht, auf die vorhin angesprochene Istraga poturica, die legendäre Vernichtung der „,vertürkten“ Bewohner Montenegros zu Beginn des 18. Jahrhunderts, als epochal gedachtes Ereignis näher einzugehen. Dabei wird ersicht-

32 Ebd., 45.

33 Bojović 1992, 189.

34 Minkov 2004, 89; zum Verweis auf das islamische Familienrecht im oben angefuihrten Zusammenhang ebd.

35 Dašić 1980, 42, hier Anm. 104. 
lich, dass eine prinzipielle „ewige Feindschaft“ zwischen der christlichen und der muslimischen Bevölkerung dieses Gebiets genauso ein Mythos war und ist wie die gerade angesprochene angeblich großangelegte Vernichtungs- bzw. Vertreibungsaktion aus Veranlassung des Bischofs Danilo. Dass eine solche in der Tat stattgefunden hat, behaupten - trotz berechtigter Kritik an dieser These seitens der Vertreter auch der eigenen Zunft - noch heute einige montenegrinische Historiker und Publizisten. ${ }^{36}$ Dabei gehen sie methodisch unkritisch vor und verfangen sich dabei in einer Fehlinterpretation und Überdehnung der historischen Beweiskraft bestimmter Ereignisschilderungen in der serbisch-montenegrinischen heldischen Volksepik, auf die auch Njegošs Bergkranz gründet. Demgegenüber hat der bekannte montenegrinische Ethnologe Jovan Erdeljanović im Rahmen seiner Forschungsreisen 1910-11 unter Befragung der örtlichen Bevölkerung herausgefunden, dass jenes Ereignis mitnichten in der historischen Erinnerung der Befragten verankert geblieben ist. ${ }^{37}$ Gerade dies hätte man bei einem derartigen von der Volksepik und der von ihr inspirierten wissenschaftlichen und nichtwissenschaftlichen Publizistik in Serbien und Montenegro als epochal eingestuften Ereignis eigentlich erwarten müssen. Auch ist keinerlei Hinweis auf das Geschehnis in der bekannten historischen Abhandlung des Fürstbischofs Vasilije von 1754 sowie in M. Medakovićs berühmter Geschichte Montenegros von 1850 vorhanden. Selbst wenn man großzügig über die zweifelhafte Authentizität des einzigen existierenden Quellenbelegs, nämlich einer nur noch als Abschrift erhaltenen Schrift, angeblich aus der Feder des Bischofs Danilo selbst, hinwegsieht, macht doch der Text des Dokuments Folgendes klar: Der Autor, der sich als Bischof Danilo einführt, stellt die berühmte „Vernichtung der Konvertiten“ lediglich als mehrfach gescheiterte Racheaktion nach der eigenen Inhaftierung und Malträtierung durch den örtlichen paşa dar. Die Masse der Montenegriner habe sich einem von ihm befohlenen gewaltsamen Vorgehen gegen die ,poturice" wiederholt konsequent widersetzt. Danilo versuchte, dem Text zufolge, deshalb, die Bevölkerung dazu zu bewegen, wenigstens diejenigen Konvertiten, die sich einer Rückkehr zum Christentum verweigerten, zu töten. Er habe es aber lediglich geschafft, acht Personen zu mobilisieren, die die betroffenen Muslime nur in dem kleinen Gebiet des Cetinje-Stammes sowie der Ćeklići am Ende tatsächlich umgebracht hätten. ${ }^{38}$ Auch wer die Echtheit dieser Quelle nicht in Zweifel zieht, muss also die Aktion gegen die „poturice“ als lokal sehr begrenztes, im Großen und Ganzen fehlgeschlagenes Unternehmen einstufen, das

36 Aus der neuesten Forschung ist zu nennen: Šćepanović 2002, 136. Vgl. hierzu auch die serbische pseudowissenschaftliche Publizistik, etwa Srpska diaspora 2001, 1. Aus der älteren Literatur s. besonders: Jovanović 1947, $91 \mathrm{ff}$.

37 Stanojević 1955, 34.

38 Ebd., 31,32, 36, 37. 
nichts mit einer Großtat von epochaler Bedeutung, d.h. einer grundlegenden endgültigen Abrechnung mit den Islam-Konvertiten in Montenegro, zu tun hat, wie sie die Heldenepik spiegelt.

$\mathrm{Ob}$ man nun die Frage der Authentizität dieses Dokuments stellt oder nicht, so muss doch die darin deutlich zum Ausdruck gebrachte Unwilligkeit der örtlichen Bevölkerung und der lokalen Führer, sich an einer gemeinsamen Großaktion gegen die ,poturice“ zu beteiligen, angesichts des vorhin Gesagten keinesfalls verwundern. Die Schrift wäre in jedem Fall ein weiterer Beleg nicht nur für den Primat der althergebrachten Stammesgesetze und Gebräuche über den erklärten Willen eines regierenden Bischofs, sondern vor allem auch für die mangelnde soziale und ethnische Spaltung der Stammesgesellschaft nach Glaubenskriterien. Auch die Islamisierten waren ja Mitglieder der eigenen Bruderschaft bzw. des eigenen Stammes, gegen die sich eine Gewaltanwendung, nur weil sie sich einem anderen Glauben überantwortet hatten, verbot.

Diese aus der Quelle zu gewinnenden Erkenntnisse sind meines Erachtens entscheidend für die Beurteilung der gesellschaftlichen Position der „poturice“ in Montenegro bis mindestens um die Mitte des 18. Jahrhunderts. Eine Bestätigung dieses Befundes ergibt sich im Übrigen aus den vielfältigen Formen der Zusammenarbeit, einschließlich der regen Geschäfts- und Tauschbeziehungen zwischen montenegrinischen Orthodoxen und Konvertiten bzw. deren Nachkommen im 17. und 18. Jahrhundert, wie sie die venezianischen Quellen reichlich belegen. ${ }^{39}$

Aber selbst noch 1878 waren sich nicht wenige der lokal regierenden Begs und Agas als Nachkommen der ,poturice“ im Einzelfall ihrer Nähe und stammlichen Verbundenheit mit den gleichfalls südslawischen orthodoxen Christen durchaus bewusst. Man kann sogar mit gutem Grund behaupten, dass sich diejenigen Bevölkerungsteile, die erst spät, also im 19. Jahrhundert, zum Islam konvertierten und in die Region des sancak von Novi Pazar zuwanderten, auch lange nach ihrer Umsiedlung immer noch als islamisierte Slawen, nicht als Türken, fühlten und sich ihrer Abkunft als alten montenegrinischen Stämmen der Vasojevići, Piperi, Bjelopavlići, Drobnjaci u.a. zugehörig stets eingedenk waren. ${ }^{40}$

Als Beispiel hierfür kann die vorhin bereits erwähnte muslimische Elitenfamilie der Mušovići dienen. In dem Jahr der Eigenstaatlichkeit Montenegros 1878 hielt Lutvi-beg Mušović anlässlich eines Aufenthalts in Sarajevo in seiner Eigenschaft als Führer einer Delegation der Nikšićer Muslime eine bemerkenswerte Rede, die folgendermaßen begann:

39 Ebd., 40, 41.

40 Mušović 1979, 72. 
Brüder Türken und Serben! Mir hat der erlauchte Fürst Nikola gesagt, dass wir nach Blut und Sprache Brüder sind und dass unsere Alten eins waren, und so ist es auch, wie wir alle wissen. Aber nachdem uns die Türken erobert, unseren Glauben geteilt und bewirkt haben, dass wir uns einander entfremdet und unseren alten Ruhm vergessen haben, so ist einer dem anderen zum Henker geworden und wir haben uns gegenseitig gemordet und gemetzelt, gehasst und vernichtet über diese drei Jahrhunderte. Aber Gott hat vielleicht befohlen, dass es so sein soll, wegen gewisser Sünden unserer Alten... O, wenn nur unsere Alten - sprach der erlauchte Fürst Nikola - einmütig gewesen wären, hätten die Vögel wegen unseres [...] einblütigen Volkes anders gesungen. ${ }^{41}$

Diese anrührenden Worte klingen wie die Beschwörung von Einigkeit und Brüderlichkeit zwischen den montenegrinischen Christen und Muslimen nach vielfach erfahrenem Leid auf beiden Seiten. Mit dem Wort „Türken“, das Mušović direkt zu Beginn der Rede verwendet, sind natürlich die Nachkommen der einstigen Konvertiten, also der „,poturice“, gemeint, ein lange eingeführter Sprachgebrauch, der in der Rigorosität der Gleichsetzung mit den ethnischen Türken auch das Selbstverständnis derjenigen, die schon früh zum Islam übertraten, spiegelte. ${ }^{42}$ Mit der zweiten Nennung des Begriffs „Türken“ sind indessen eindeutig die ethnischen Türken gemeint. Auf diese gleichsetzende Semantik und das dahinterstehende Denken hat der exklusive Nationalismus in Montenegro in der zweiten Hälfte des 19. Jahrhunderts bis zur Niederlage im Ersten Weltkrieg instrumentalisierend zugreifen können. Allerdings konnte er nie alle montenegrinischen Gesellschaften einbinden und nach ethnisierten Glaubenskriterien spalten. Als Beispiel seien die christlichen Çiftlik-Bauern und ihre muslimischen, oft nicht minder armen Berufsgenossen im Raum Rožaje und im Bihor-Gebiet angeführt, also Gegenden, die erst nach den Balkankriegen dem montenegrinischen Staat angegliedert wurden: 1853 kämpften beide Gruppen gemeinsam mit den Serbisch-Orthodoxen gegen die lokal Mächtigen, besonders gegen die Grundbesitzer. Es war eine Revolte gegen unerträgliche feudale Lasten (Abgaben). Der Widerstand gegen diese führte in der zweiten Hälfte des 19. Jahrhunderts überhaupt häufig zu Rebellenbewegungen christlicher und muslimischer Bauern in der gesamten Region Novi Pazar-Tutin gegen die muslimischen Çiftlik-Eigner. ${ }^{43}$

Damit soll keineswegs gesagt werden, dass es im Montenegro des 19. Jahrhunderts unter dem Einfluss des heraufziehenden Nationalismus keine grundsätzlichen gesellschaftlichen Antagonismen und Spaltungstendenzen zwischen den beiden Religionsgruppen gegeben hätte, noch dass diese zu keinen bewaffneten Auseinandersetzungen oder auch längeren Kampfhandlungen führten.

41 Ebd., Mušović 1981b, 91, 92.

$42 \mathrm{Zu}$ diesem Selbstverständnis der frühen Konvertiten vgl. die Ausführungen oben S. 273, 274 sowie auch Mušović 1979, 72.

43 Mušović 1979, 82. 
Wenn wir uns die Zeitzeugnisse ansehen, so wird schnell klar, dass die vielzitierte, oben erwähnte Istraga poturica Njegošs tatsächlich vor allem im 19. und 20., eben nicht im 17. Jahrhundert historisch fassbar wird, wenn auch nicht als einmaliges Ereignis, sondern eher als Prozess, d.h. als eine Abfolge von mehr oder weniger gewaltsamen Geschehnissen: Um die Mitte des 19. Jahrhunderts, in der Zeit der Kriege Danilos I. mit dem Großherrn, spitzte sich das Wüten der montenegrinischen Stämme gegen die mächtigen Begs und Agas zu. Die islamisierte Sippe der Mušovići bietet auch hier ein gutes Beispiel. Nach der 1858 erreichten internationalen Festlegung der montenegrinischen Autonomiegrenzen brachen heftige Kämpfe gegen führende Exponenten der genannten Familie in und um die Stadt Kolašin aus. Die Befestigungsanlagen der Mušovići gingen dabei in Flammen auf, und die gesamte muslimische örtliche Bevölkerung floh aus der Stadt. Ein Teil siedelte sich in den Dörfern des unteren Kolašin-Gebiets an, auch in Vraneš und Šahović, ein anderer Teil zog weiter nach Süden. 186062 loderten die Kämpfe erneut auf, danach insbesondere in den Jahren 1875-78. Der 1860 erfolgte Angriff von 700 christlichen Kämpfern auf den Stammsitz der Mušovići, Stričina, der nur einige Stunden dauerte, brachte 70 Angreifern den Tod, die muslimische Gegenseite hatte hingegen den dreifachen Blutzoll zu zahlen. 1861-77 fielen zwei Agas sowie ein Stadtkommandant der lokal regierenden Familie, ferner 1877 nach schweren Verlusten der Muslime auch die gesamte Besatzung der Festung Kolašin aufständischen montenegrinischen čete, also christlichen Kampfgruppen, zum Opfer. Ständige Konflikte zwischen den beiden um den Grenzfluss Tara siedelnden Bevölkerungsgruppen im unteren Kolašin-Gebiet verschärften die bedrohte Lage gerade der Muslime beträchtlich, bis die noch lebenden Mušovići und die gesamte muslimische Bevölkerung des Gebiets im Jahr 1924, also am Ende der bürgerkriegsähnlichen Unruhen im Lande $^{44}$, endgültig in die Türkei emigrierten. ${ }^{45}$

Allerdings muss die eskalierende Gewalt zwischen der islamisierten Bevölkerung und den christlichen Montenegrinern in der zweiten Hälfte des 19. Jahrhunderts in ihren Beweggründen differenziert betrachtet werden. Wie schon aufgrund des Gesagten vermutet werden kann, ist sie nämlich nicht allein als Folge des mobilisierenden Einflusses des aufkommenden montenegrinischen Nationalismus zu deuten. Bevor in den Jahren zwischen 1877-82 391 von 410

44 Bis in dieses Jahr zogen sich die bewaffneten Auseinandersetzungen um den Status Montenegros hin. Es ging um einen Kleinkrieg zwischen den Verfechtern der Königstreuen, die als Anhänger des Exilkönigs Nikola die bis 1918 herrschende einheimische PetrovićDynastie wiederherzustellen wünschten, und den Anhängern einer vollständigen Inkorporierung Montenegros in den serbischen Staat. Letztere wurden vom im Lande befindlichen serbischen Militär unterstützt. Dazu Näheres bei Rastoder 2003, 131, 132.

Mušović 1981b, 95-97. 
muslimischen Familien der Stadt Nikšić wegen der erlebten Gewalt und der sich radikal wandelnden politischen Verhältnisse das Land verließen ${ }^{46}$, war die Mehrheit der christlichen Montenegriner für einen solchen Abzug, Fürst Nikola jedoch dagegen. Er wollte den muslimischen Bevölkerungsteil, wegen der gemeinsamen Abkunft, die er betonte, möglichst im Lande halten. ${ }^{47}$ Diese Linie verfolgte auch die nunmehr königliche Regierung im Frühjahr 1914, als ein paar tausend muslimische Familien aus Nordmontenegro abwanderten. ${ }^{48}$ Es ist die historische Erinnerung an die gemeinsamen Wurzeln der christlichen wie auch der islamisierten südslawisch-montenegrinischen Bevölkerung, an die König Nikola mit Nachdruck anknüpfte. Aber auch so mancher montenegrinischer četnik sah sich veranlasst, nicht wahllos „Türken“ zu jagen und zu töten, sondern, wie es in zeitgenössischen Quellen heißt, sich die besten „Köpfe“ zu holen. Hier spiegelte sich traditionelles agonales Stammesdenken, denn es kam darauf an, denjenigen Gegner zu töten, der am angesehensten war und den besten Ruf als heldenhafter Kämpfer genoss, um des eigenen Ruhmes und der Erhöhung des Ansehens der eigenen Sippe willen. Die führenden Köpfe der auch bei den christlichen Sippen trotz aller Gegnerschaft hochangesehenen islamisierten Familie Mušović geben auch in dieser Beziehung gute Beispiele ab. ${ }^{49}$ Nationaler bzw. nationalistischer Hass spielten vor diesem Hintergrund letztlich eine nur nebengeordnete Rolle, oder anders ausgedrückt: Die kognitiven und affektiven nationalistischen Dispositionen und Ausbrüche wurden von der traditionellen Stammesethik gebremst und sozusagen „eingeholt“. Selbstverständlich änderte dies nichts an der faktisch zu konstatierenden direkten oder indirekten, oft sehr brutalen Gewalt unter beiden Gruppen.

Aus dem hier Dargelegten ergibt sich, dass das Problem der montenegrinischen „poturice“ und deren Nachkommen als spezieller muslimisch-südslawischer Bevölkerungsschicht bezüglich ihrer gesellschaftlichen, wirtschaftlichen und politischen Position und Bedeutung in der älteren und jüngeren Geschichte sehr diffizil und vielschichtig ist. Es wird noch weiterer detaillierter wissenschaftlicher Analysen, ggf. auch Feldforschungen vor $\mathrm{Ort}^{50}$, bedürfen, um unser bisher gewonnenes Bild zu vervollständigen. Meine Ausführungen sollen in diesem Sinne ein Anstoß für die weitere Erforschung dieses bisher viel zu wenig beachteten Gegenstands sein.

46 Vgl. Mušović 1979, 89.

47 Mušović 1981b, 94.

48 Babić 1973, 166, 167.

49 Mušović 1981b, 94, 95.

50 Etwa repräsentative Befragungen älterer Montenegriner bezüglich ihrer Familiengeschichten. 


\section{Literaturverzeichnis}

Babić, Branko, 1973: „Migracije u novooslobođenim krajevima Crne Gore 1912-1915“, in: Jugoslovenski istorijski časopis 12(1973), H. 3-4, 163-170.

Babinger, Franz, 1959: „Die Osmanen auf dem Balkan“, in: Völker und Kulturen Südosteuropas. Kulturhistorische Beiträge. München (= Südosteuropa. Schriftenreihe der Südosteuropa-Gesellschaft; Bd. 1. Hrsg. von Wilhelm Gülich), 199-210.

Bartl, P., 1974: „Danilo“, in: Biographisches Lexikon Südosteuropas. Bd. 1 (A-P). München, $370-371$.

Bojović, Jovan P., 1992: „Neki podaci vojvode Marka Miljanova Popovića o običajima u Kučima i neki dokumenti o Kučima u Bogišićevom arhivu u Cavtatu“, in: Istorijski zapisi LXV, 1992/1-4, 187-201.

Clewing, Konrad, 1999: „Muslime“, in: Roth, Klaus (Hrsg.): Geschichte Ostmittel- und Südosteuropas. (= Studienhandbuch östliches Europa; 1). Köln u.a., 482-485.

Dašić, Miomir, 1980: „Feodalni odnosi i etnička kretanja u gornjem Polimlju u XVIII i prvoj polovini XIX vijeka“, in: Istorijski zapisi XXXIII (LIII), 1980/3, 5-63.

Dašić, Miomir, 1999: „Montenegro bis 1918“, in: Melčić, Dunja (Hrsg.): Der Jugoslawien-Krieg. Handbuch zu Vorgeschichte, Verlauf und Konsequenzen. Opladen, 109-119.

Deretić, Jovan, 1996: Kompozicija Gorskog Vijenca. Podgorica.

Dragović, M., 1931: Poturčenjaci u Crnoj Gori. Cetinje.

Dragović, M., 1935: Istorija Crne Gore. Dio 1. Podgorica.

Hadrijahić, Muhamed, 1980: „Odnosi bosanskog Ejaleta sa Crnom Gorom u XVIII vijeku“", in: Istorijski zapisi XXII (LIII), 1980/3, 85-97.

Istorija Crne Gore. Knjiga treća, od početka XVI do kraja XVIII vijeka. 1975. Tom I. Predsjednik redakcije: Milinko Durović. Titograd.

Jovanović, Jagoš, 1947: Stvaranje crnogorske države i razvoj crnogorske nacionalnosti. Istorija Crne Gore od početka VIII vijeka do 1918 godine. Cetinje.

Kalezić, Slobodan, 1987: „Zajednički motivi u kolima Njegoševa ,Gorskog Vijenca u narodnim pjesama iz Vukovih i drugih zbirki“", in: Milović, Jevto M. (ur.): Vuk St. Karadzicic i Crna Gora. Radovi sa naučnog skupa, Titograd, 16. i 17. aprila 1987. Titograd, 123-137.

Medenica, Radosav, 1964: „Gorski vijenac - narodna svetkovina“, in: Vukmanović, Jovan (Glavni urednik): Rad X-og Kongresa Saveza Folklorista Jugoslavije na Cetinju 1963. godine. Cetinje, 125-135.

Minkov, Anton, 2004: Conversion to Islam in the Balkans. Kisve Bahası Petitions and Ottoman Social Life, 1670-1730 (= The Ottoman Empire and Its Heritage. Politics, Society and Economy; Vol. 30). Leiden, Boston.

Mušović, Ejup, 1979: Etnički procesi i etnička struktura stanovništva Novog Pazara. Urednik Petar Vlahović (= Srpska Akademija Nauka i Umjetnosti. Etnografski Institut. Posebna Izdanja; knjiga 19). Beograd.

Mušović, Ejup, 1981a: „Islamizacija u novopazarskom sandžaku“, in: Istorijski zapisi XXXIV (LIV), 1981/3-4, 5-19. 
Mušović, Ejup, 1981b: „Mušovići“, in: Istorijski zapisi XXXIV (LIV), 1981/2, 87-102.

Pejović, Đoko D., 1979: „Iz istorije područja Pljevalja i Bijelog Polja od kraja XVIII do sredine XIX vijeka“, in: Istorijski zapisi XXXII (LII), knj. XLI, 1979/4, 71-96.

„poturica“, in: Rječnik hrvatskoga ili srpskoga jezika na svijet izdaje Hrvatska Akademija Znanosti i Umjetnosti. Obradio T. Maretić. Dio XI: Pomrtnik - Prikladańe. U Zagrebu 1935, 222.

„poturica“, in: Srpski rječnik istumačen njemačkijem i latinskijem riječima. Skupio ga i na svijet izdao Vuk St. Karadžić. Treće (državno) izdanje, ispravljeno i umnoženo, u Beogradu 1898, 575.

„poturica“, in: Wörterbuch der serbokroatischen und der deutschen Sprache. Zweiter Teil: Serbokroatisch - Deutsch von Dr. phil. Swetomir Ristitsch und Jowan Kangrga. Bel$\operatorname{grad} 1928,732$.

Rastoder, Šerbo, 2003: „A Short Review of the History of Montenegro“, in: Bieber, Florian (ed.): Montenegro in Transition. Problems of Identity and Statehood. BadenBaden, 107-137.

Rizvić, Muhsin, 1985: Kroz „Gorski Vijenac“. Interpretacija i tekstualno-komparativna studija o strukturi. Sarajevo.

Šćepanović, Žarko, 2002: Kratka istorija Crne Gore, od najstarijih vremena do 1796. godine. Podgorica.

Srpska diaspora. Internet Novine Serbske, uz diasporu od 2001. godine; http://www. srpskadiaspora.info/vest.asp?id=5072.

Stanojević, Gligor, 1955: Crna Gora u doba vladike Danila. Crne Gore.

Vasić, Milan, 1985: Der Islamisierungsprozess auf der Balkanhalbinsel. Graz.

Vasojevićki zakon od dvanaest točaka, s komentarom D-ra Ilije Jelića. Beograd 1929.

Zirojević, Olga, 2003: Islamizacija na južnoslovenskom prostoru. Dvoverje (= Etnološka biblioteka ; knj. 10), Beograd. 
